\title{
PEER-TO-PEER PROCESS INTEGRATION IN VIRTUAL ENGINEERING ORGANIZATIONS
}

\author{
Eva-Maria Kern \\ Production Management; Technical University of Hamburg, GERMANY; e.kern@tuhh.de
}

Axel Hahn, Alf Benger

Business Information Systems, University of Oldenburg, GERMANY; hahn|benger@wi-ol.de

\begin{abstract}
Virtual organizations are temporary networks built up by independent companies in order to achieve a certain business purpose. The resulting flexible and dynamic virtual organizational structures require the easy and seamless integration of new partners in order to create an efficient cooperation. The introduced approach has the main aim to support flexible process integration by using a P2P architecture for virtual engineering organizations in shipbuilding industries. The approach uses a semantic process description model to support the set up and execution of the process. The P2P approach is extended by the concept of communication and collaboration policies to give dedicated partners the required control of the virtual organization.
\end{abstract}

\section{INTRODUCTION}

Although the concept of virtual organization is widely accepted as the organizational structure of future organizations (Hales/Baker 2000), the practical adaptation still lacks a proper IT support (Bajwa/Lewis 2002). Especially in engineering environments there are opposed requirements, on the one hand the engineering networks are temporary and should be flexible and on the other hand there is the requirement for deep integration. This contradiction is even true in the Shipbuilding Industry, where the shipyard in the center of the development process subcontracts a large number of development partners. A visible trend of the development process is that it is carried out as an IT supported collaborative process between various experts from different companies setting up a virtual engineering organization (Gronau 2003). These virtual teams of distributed participants - working on an integrated product development project - are virtually co-located and coordinate their efforts virtually. Especially for the creation of spontaneous engineering networks an efficient process integration procedure is necessary to realize an easy and seamless integration of new partners, because the partner specific processes have to be coordinated to create a common process. The IT architectures that are currently used to support virtual engineering organizations show a conceptual gap between their technological and organizational structure (Orlikowski/Gash 1994). To support the engineering process more efficiently the authors propose an IT architecture that is based on a Peer-to-Peer network. 


\section{VIRTUAL ENGINEERING ORGANIZATIONS IN THE SHIPBUILDING INDUSTRY}

A challenging example for the management of virtual engineering organizations is the Shipbuilding Industry. In its very complex distributed engineering processes more than $75 \%$ of the creation of value is performed by numerous suppliers. Besides material, component and system suppliers also engineering companies can be used to support the shipyard with construction performance in various extents. Depending on the progress of the project the active participants of the virtual engineering organization may change. The engineering collaboration is characterized by a large number of various companies, which differ very strongly in their size, their structures, their competencies, tasks and importance for the engineering process.

In Germany the shipyards concentrate on the development and production of small series or even uniques. So the standardization potential of the engineering processes is very low and for every shipbuilding project changing partner constellations have to be taken into account. Usually the embedded organizations do not only join a single shipbuilding project, but also participate in several engineering collaborations, also with different shipyards, at the same time. Especially for small and medium enterprises (SME) the participation in different virtual organizations is a major organizational and technical challenge because every shipyard has its specific requirements for collaboration, e.g. the use of certain CAD-Systems.

\subsection{Characteristics and Challenges of Distributed Engineering Processes in the Shipbuilding Industry}

The shipyard in its position as main contractor is the center of the engineering process and responsible for the success of the whole project. One of its main tasks, beside constructive and planning performance, system integration and final production, is project management. Therefore it holds the most powerful and determining position in the virtual engineering organization. Depending on the development target, e.g. type of ship, it negotiates with the suppliers and finally selects them, partially based on the demand of the customer. During the whole project it coordinates the partners, controls their performance and integrates their contributions. Normally there is no direct communication about important details between the suppliers, everything has to be negotiated and adjusted directly with the shipyard, which wants to keep track of the projects progress. It holds continuous control over the communication processes and is responsible to distribute necessary information among the partners.

Due to the product "ship" the distributed engineering process has a very high complexity. Big volumes of data and information have to be exchanged between the engineering partners. The interdependency between the specific tasks of the main suppliers and the shipyard is very high. This means that a change in one system can possibly affect several other systems or even the whole construction of the ship. Therefore, the well directed communication of changes is an important challenge in project management, especially because there are a number of mostly constructive changes on the product due to customer wishes or knowledge increase, also in an advanced status of the project. 
Based on an actual empirical study (Kersten/Kern/Zink 2002) the main obstacles of nowadays engineering processes in shipbuilding are as well of organizational as of technical nature:

- The partners have a different understanding of the over-all process.

- Information demands of the partners are not transparent. So they do not get the information in the quality they need at the right time. This concerns standard communication as well as change management, which means there is a lack of communication guidelines and policies.

- The IT Infrastructures of the partners are very heterogeneous. The data and information transfer is problematic due to different systems and versions.

- There are no appropriate IT-Tools for partner integration. Engineering platforms, which are under development in several research projects (Lukas 2001), require a high effort of implementation and do mainly not support flexible partner integration.

\subsection{Flexible Process Integration}

The obstacles mentioned above show that an essential challenge in the collaboration is the target-oriented coordination of the partners and the design of the information flows between them. As Figure 1 depicts, engineering partners with different internal structures and processes have to be integrated in a common over-all process. Flexible partner integration means that the engineering partners can easily enter and leave the virtual engineering organization. This should be handled locally without affecting the whole virtual organizational structure or the company specific structures.

An appropriate approach for the Shipbuilding Industry has on one hand to support the central role of the shipyard, on the other hand to keep the organizational and technical integration threshold for the engineering partners as low as possible.

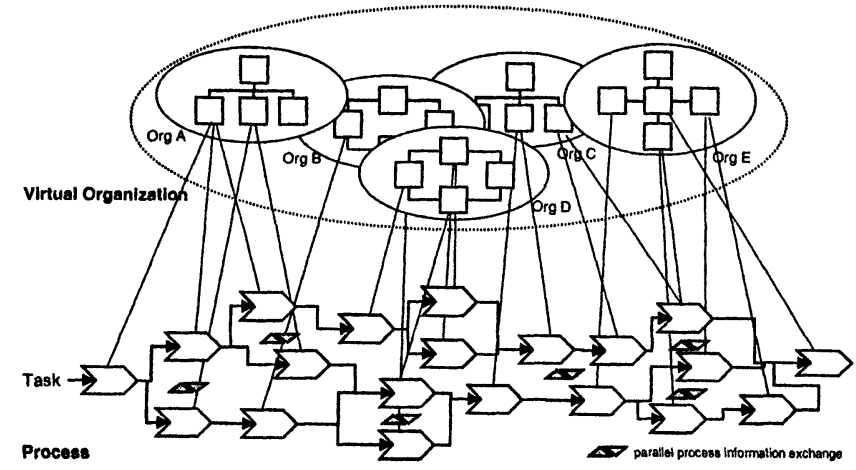

Figure 1 - Assigning tasks to different organizational structures

The flexible process integration has both technical and organizational challenges:

- Technical aspects of the integration: The technical integration is one of the key challenges, because existing standards and integration technologies still require high integration efforts or are lacking in accuracy or compatibility. The 
deployment of a central IT platform for temporary engineering organizations is too timely and costly for very dynamically changing organizations.

- Organizational aspects of the integration: Flexible process integration can be realized if the engineering partners internally follow their own development processes and decide themselves about internal task priorization and the assignment of engineering resources. Externally they offer clear and defined interfaces for collaboration according to the requirements of their partners. Process specifications should describe the generated business and development value, communication points and their semantic as well as the required information exchange. This requires a proper interface and process description to set up the co-operation inside the virtual organization.

The main aim of the approach described in this paper is to support flexible process integration. For its realization the following chapter introduces a concept that is based on a Peer-to-Peer network.

\section{PEER-TO-PEER PROCESS INTEGRATION}

Peer-to-Peer (P2P) networks offer the ideal infrastructure for an easy integration of virtual organizations by providing a flexible co-operation infrastructure that supports the communication and coordination aspects required for virtual engineering organizations.

\subsection{Peer-to-Peer Networks}

A P2P network architecture consists of a number of nodes (or organizational units) that have a high degree of autonomy. These nodes communicate directly to each other without going through a central server or hub. Data and information are stored locally, thus the nodes maintain the ownership and control over the shared resources. The P2P approach is suitable for virtual engineering organizations due to the following system characteristics (Milojicic et al 2002):

- Autonomy: The engineering partners often prefer to put all data in a local storage, working on those data locally, and share the data only as needed.

- Privacy/Access control: The engineering partner can decide which information to share. Internals of a node like in-house processes etc. can be hidden.

- Flexible configuration: The engineering groups can enter or leave the network at any given point of time. Private or public groups can be easily configured without the need to install additional software applications or components.

- Cost sharing/reduction: In most cases it is sufficient to install an additional software component on existing information systems.

- Resource aggregation and interoperability: A decentralized approach leads naturally to resource aggregation. Each engineering partner contributes certain resources (engineers, tools, etc.) to the engineering project.

\subsection{Process Integration}

To set up a common development process the sub-processes at the development units have to be identified. In this conception the internal sub-processes are modeled 
as black boxes named building blocks. During the process configuration the building blocks are instantiated to the development process.

The usage of process models like NIST's Process specification Language (PSL) (Schlenoff et al. 2000) or from the WFMC (Workflow Management Coalition 1999) would provide a level of detail about the internal processes of an organizational unit that is not required by participating engineering teams.

Ankolekar et al. (2001) introduced DAML-S, a DAML+OIL ontology, to describe the capabilities of Web Services. DAML-S provides semantic services descriptions in three conceptual areas: the profile, the process model, and the grounding. The profile describes what the service does, the process model tells how the service works and the grounding specifies how the service is used. DAML-S slightly adapted - can also be used to describe the building blocks. The profile information is used for the identification of a proper building block to set up and run the development process. The process model and grounding specification is used for process integration. Due to the rich semantic expressive power DAML-S is well suited to describe the processes, development resources and potential value generated by the unit while hiding the process internals from the public.

By using the P2P network DAML-S specifications can be searched and retrieved (Bryson et al. 2003) to set up the develop process.

An example task is a Finite Element Analysis (FEA) of the hull structure of the ship. The ontology is used to classify the task. Required input is a 3D model represented in a grid. As a precondition the design has to be approved by a review process task. The output is a stress model and information of the compliance to the related standards of the ship class. The effect of this task may be the initialization of a redesign.

\subsection{Peer-to-Peer Approach to Support Virtual Engineering Organizations in the Shipbuilding Industry}

One of the characteristics in the shipbuilding industry is the fact that the shipyard dominates the engineering network and therefore wants to take control of the communication between the engineering partners. Usually P2P approaches imply equal partners and decentralized communication processes. So a concept of communication and cooperation policies is introduced, which allows the shipyard to control the cooperation in the P2P network by defining these policies for every participating engineering partner or for predefined groups (see fig. 2) assigned to specific development tasks. So the shipyard can control who has access to which information, assure that it is involved in all important interactions, but is unburdened from communication processes which only effect specific partners. The P2P approach guarantees low cost for the IT infrastructure because the shipyard does not has to maintain a central IT platform for the participating engineering partners.

The following three main co-operation policy types were identified by the authors:

- Open Communication: Information can be accessed and modified by all participating engineering partners. [fig. 2(a)]

- Private Communication: Private communication takes place between a predefined number of engineering partners. Engineering teams will be invited by 
the shipyards to join a group. This can be used e.g. for change management support. [fig. 2(b)]

- One-to-One Communication: The communication takes only place between the shipyard and the engineering partner. [fig. 2(c)]

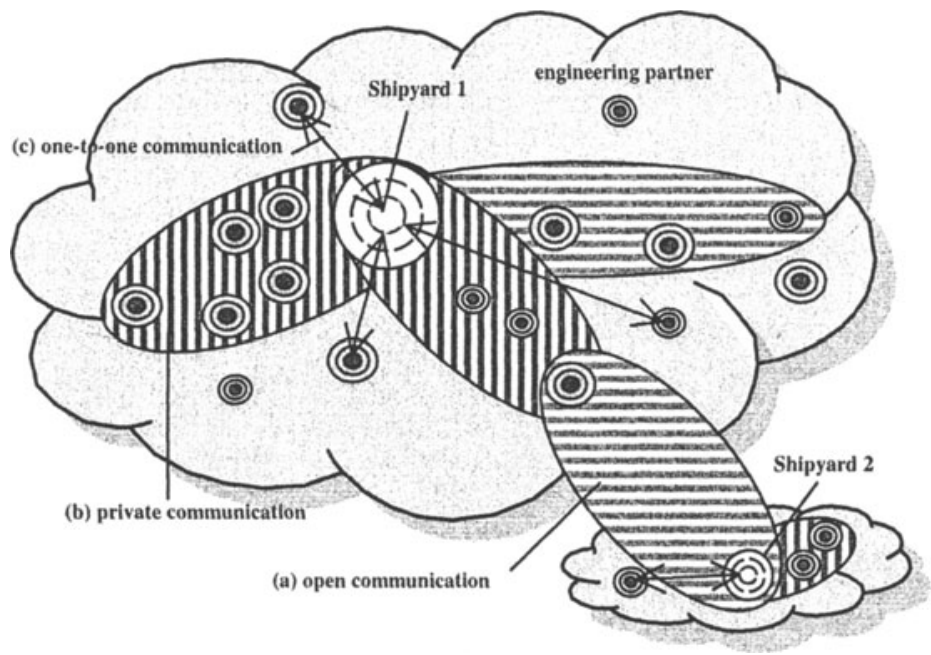

Figure 2 - Shipbuilding Ecosystem

The policies include rules to set up and run the development processes by providing guidelines for the partner selection, assignment and delivery of engineering tasks. They also include the definition of file formats and standards, process interaction protocols, quality issues as well as security and confidentiality policies.

\subsection{Network Architecture}

For the implementation the authors propose an architecture based on a standard P2P framework, e.g. the JXTA framework by Sun Microsystems (Gong 2001). The JXTA framework is a set of open, generalized Peer-to-Peer protocols that allow devices to communicate and collaborate through a connection network. The framework provides also protocols such as a decentralized resource discovery, asynchronous point-to-point messaging system and protocols to form open groups. A peer in this framework is a software component that runs the basic JXTA protocols and agrees on a common set of rules to publish, share and access resources (Traversat et al 2001).

The JXTA architecture has three main layers (as shown in fig. 3). The first layer, (JXTA core) handles information exchange. The second layer provides standard JXTA services and the X-Peer services introduced by the authors in section 3.5.

The architecture can also be extended by collaborative virtual engineering applications in the third layer that can extend the approach introduced in this paper. 


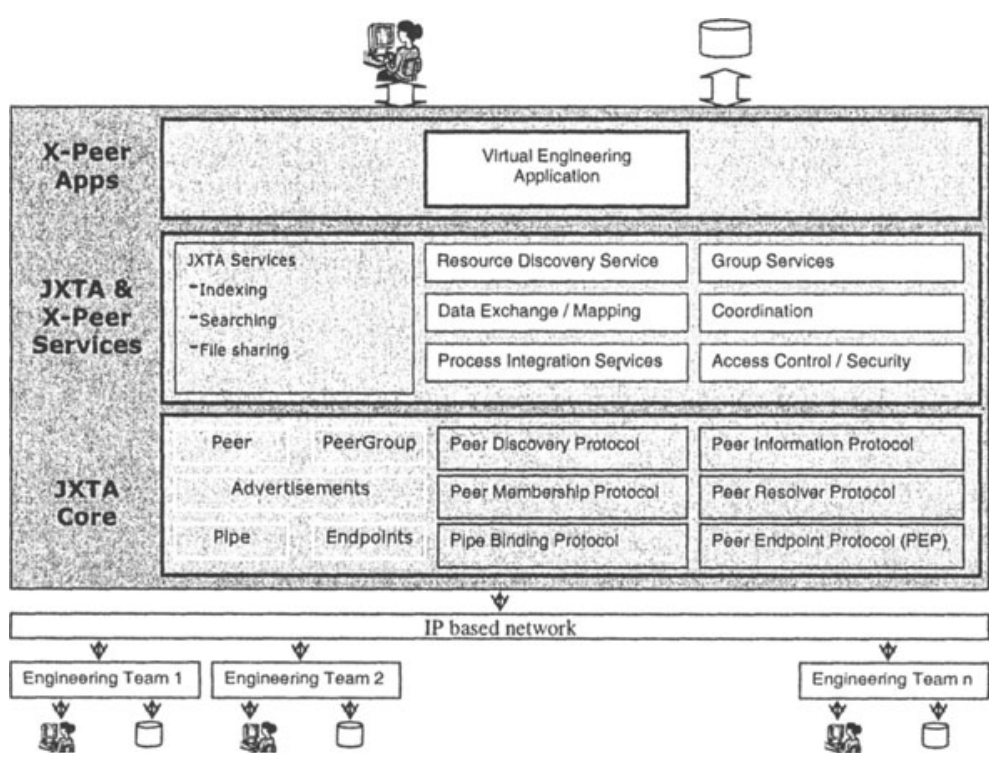

Figure 3 - X-Peer Services

\subsection{X-Peer Services}

To allow easy process integration, the following X-Peer Services are implemented:

Resource Discovery Services: In the Peer-to-Peer network the semantic specification of the process building blocks can be searched and retrieved. The selection of the appropriate building block is not done fully automatic. The engineer uses his knowledge about the actual development situation. Suitable building blocks are identified by their semantic description and the specification of the input/output/precondition/effect specification expressed with DAML-S (Ankolekar, et al., 2001). For the identification of the building blocks the cooperation polices from the shipyard are obeyed. The input and precondition specification are examined to identify whether all required information for the process execution are available (Narayanan/McIllraith, 2002). Input and output specifications control the information/file exchange between the partners. Output and effects specifications are used to identify following process activities. Following the example given in chapter 3.2 the FEA Service offered by an engineering company can be identified by the classification ontology by using the process specification and the applicability of the task in the current design situation can be checked.

Process Integration Services: The identified process building blocks are used to configure the local process management. In the example in the engineering company the internal FEA process is initiated by the system. The output is passed back to the requesting peer. If a SME has no appropriate system the engineer can manually follow the required process specification provided.

Data Exchange / Mapping Services: Regarding the agreement about file exchange standards, quality requirements and communication policies the engineering information exchange is controlled. Available filters for file 
transformation are used automatically (e.g. DXF to a STEP model for FEA with a specified grid size).

In addition to the specific services, same additional services will be provided, such as group services, e.g. shared workspaces; coordination services, e.g. tools for project management and access control services to keep track of the distribution of information.

\section{CONCLUSION}

The distributed engineering processes of shipbuilding industry require a broad support of communication and collaboration. An appropriate IT solution has to consider the dominating and controlling role of the shipyard and to guarantee flexible, dynamic partner integration with low implementation efforts. Due to these requirements a Peer-to-Peer approach was chosen, providing communication, integration and collaboration services. The authors extend the P2P paradigm by introducing policies that can be defined by dominating peers to control the collaboration processes between the partners.

\section{REFERENCES}

1. Ankolekar A, Burstein M, Hobbs JR, Lassila O, Martin DL, Mcllraith SA, Narayanan S, Paolucci M, Payne T, Sycara K, Zeng H. DAML-S Semantic Markup for Web Services, Proc. Of the First Semantic Web Working Symposium (SWWS '01), Stanford, The SAML Services Coalition, 2001.

2. Bajwa DS, Lewis LF. Current Status of Information Technologies Used in Support of Task-Oriented Collaboration, Proceedings of the 35th Hawaii International Conference on System Sciences, 2002.

3. Bryson J, Martin D, Mcllraith SA, Stein LA. Agent-Based Composite Services in DAML-S: the Behavior-Oriented Design of an Intelligent Semantic Web, in Zhong, Liu, J. Yao, Y. (Eds) Web Intelligence, Springer, 2003.

4. Milojicic DJ, Kalogeraki V, Lukose R, Nagaraja K, Pruyne J, Richard B, Rollins S, ,Xu Z. Peer-toPeer Computing. HP Laboratories Palo Alto HPL-2002-57 March 8th , 2002.

5. Gong, Li. Project JXTA: A technology overview. Technical report. Sun Microsystems Inc., 2001.

6. Gronau, Norbert. Collaborative Engineering Communities - Architecture and Integration Approaches. In M. Khosrow-Pour (ed.), Information Technology and Organizations: Trends, Issues, Challenges and Solutions. Proceedings of 2003 IRMA Conference, Philadelphia PA, USA, 2003.

7. Hales K, Baker J. Searching for the Virtual Enterprise, Bond University, Australia, Working Paper $1 / 00,2000$.

8. Kersten W, Kern EM, Zink Th. Optimierungspotenzial zwischenbetrieblicher Informationstransfer: in: Hansa - Schiffahrt-Schiffbau-Hafen - 139. Jahrgang - 2002 - Nr.4; S. 30-32, 2002.

9. Lukas, Uwe v. Enabling Cross Enterprise Collaboration with IVIP. In U. F. Baake et.al. (ed.), Engineering: The Path to Electronic Business. Proceedings of the ECEC 2001. SCS Publication: 175-183., 2001.

10. Narayanan S, McIllraith S. Simulation, Verification, and Automated Composition of Web Services, Honolulu, 2002.

11. Orlikowski WJ, Gash DC. Technological Frames. Making Sense of Information Technology in Organizations. ACM Transactions on Information Systems, April 1994.

12. Schlenoff C, Gruninger M, Tissot F, Valois J, Lubell J, Lee J. The Process Specification Language (PSL): Overview and version 1.0 specification, NISTIR 6459, National Institute of Standards and Technology, Gaithersburg, 2000.

13. Traversat B, Abdelaziz M, Duigou M., Hugly, JC, Pouyoul, E, Yeager B. Project JXTA Virtual Network. Technical report, Sun Microsystems Inc. 2001.

14. Workflow Management Coalition. Process Definition Meta-Model \& WPDL, WfMC-TC-1016-P v1.1, 1999. 